\title{
Analysis of emergency department visits for all reasons by adults with depression in the United States
}

Tyler Hill ${ }^{1,2}$, Yun Jiang ${ }^{2}$, Christopher R. Friese ${ }^{2}$, Lynae A. Darbes ${ }^{3}$, Christopher K. Blazes ${ }^{4,5}$ and Xingyu Zhang ${ }^{2^{*}}$ (D)

\begin{abstract}
Background: We aimed to characterize Emergency Department (ED) utilization and outcomes of patients with depression seeking emergency care for all reasons.

Methods: Using 2014-2016 ED data from the National Hospital Ambulatory Medical Care Survey, we investigated demographics, ED resource utilization, clinical characteristics, and disposition of patients with depression versus those without depression.

Results: Approximately 10,626,184 (11.4\%) out of 92,899,685 annual ED visits were by patients with depression. ED patients with depression were mostly non-Hispanic White (74.0\%) and were less likely to be male than patients without depression (aOR: 0.62; [95\%] Cl: 0.57-0.68). ED patients with depression were more likely to be admitted to the hospital (aOR: 1.50; Cl: 1.38-1.63) than patients without depression. Among ED patients with depression, males were more likely than females to be seeking emergency care for psychiatric reasons (OR: 2.45; 95\% Cl: 2.10-2.87)) and to present with overdose/poisoning (OR: 1.46; Cl: 1.03-2.05).

Conclusions: We described the unique demographic, socioeconomic, and clinical characteristics of ED patients with depression, using the most comprehensive, nationally representative study to date. We revealed notable gender disparities in rates and reasons for admissions. The higher hospital and ICU admission rates of ED patients with depression suggests this population requires a higher level of emergency care, for reasons that remain poorly understood.
\end{abstract}

Keywords: Depression, Emergency medicine, National characteristics, Resource utilization

\section{Background}

Depression represents an increasingly costly public health concern in the United States [1, 2]. An estimated 17.3 million adults $(7.1 \%)$ in the US had at least one major depressive episode in 2017 [3]. Along with impairments in mood and cognition, and related decrements in work and social functioning, depressive disorders are associated with substantial healthcare resource use and

\footnotetext{
* Correspondence: zhangxyu@umich.edu

${ }^{2}$ Department of Systems, Populations, and Leadership, University of Michigan School of Nursing, Room 1177, 400 North Ingalls Building, Ann Arbor, Ml 48109-5482, USA

Full list of author information is available at the end of the article
}

economic burden [1, 2]. Estimates of direct health expenditures associated with adult depression exceed $\$ 90$ billion in the US; further costs related to suicide and workplace problems were estimated to exceed $\$ 100$ billion [2].

The Emergency Department (ED) is a frequently used care setting by patients with mental disorders [4]. Nationwide data from 1992 to 2000 showed increasing ED visits for psychiatric reasons in the US, indicating patients with psychiatric diagnoses had a higher admission rate than patients without such diagnoses [4]. This analysis [4] did not specify ED visits for patients with

C C The Author(s). 2020 Open Access This article is licensed under a Creative Commons Attribution 4.0 International License, which permits use, sharing, adaptation, distribution and reproduction in any medium or format, as long as you give appropriate credit to the original author(s) and the source, provide a link to the Creative Commons licence, and indicate if changes were made. The images or other third party material in this article are included in the article's Creative Commons licence, unless indicated otherwise in a credit line to the material. If material is not included in the article's Creative Commons licence and your intended use is not permitted by statutory regulation or exceeds the permitted use, you will need to obtain permission directly from the copyright holder. To view a copy of this licence, visit http://creativecommons.org/licenses/by/4.0/. The Creative Commons Public Domain Dedication waiver (http://creativecommons.org/publicdomain/zero/1.0/) applies to the data made available in this article, unless otherwise stated in a credit line to the data. 
depression. More recent work by Ballou et al. analyzed 2 years (2006 and 2014) of national data on US ED visits for depressive symptoms, finding the rate of these visits to be greater in 2014 [5]. However, to date, data on adults with depression presenting to the ED for all reasons (i.e., not strictly for psychiatric emergencies) have received limited attention [6]. In comparison to other diseases such as cancer and diabetes, the populational characteristics of ED patients with depression and their needs for ED care and services are yet to be investigated $[7,8]$. More targeted treatment of depressed patients in the ED through evidence-based interventions may be needed. We seek to inform efforts to improve the quality of ED care delivered to patients with depression and to reduce potentially unnecessary ED use.

By means of a secondary analysis of a large, nationally representative dataset, the current study aims to estimate ED utilization by patients with depression, describe the clinical presentation of patients with depression in the ED setting, and examine factors associated with clinical outcomes and resource utilization in this population.

\section{Methods}

This is a cross-sectional study of ED data obtained from a multiyear, nationally representative survey carried out in the US. This study used preexisting, deidentified data and thus was categorized as exempt by the University of Michigan's Institutional Review Board.

\section{Study population}

The study population consisted of all adult patients (age $\geq$ 18 years) $(N=42,832$; Weighted $N=278,699,057)$ in the National Hospital Ambulatory Medical Care Survey Emergency Department Subfile (NHAMCS-ED) from 2014 to 2016 [9]. NHAMCS-ED is a nationally representative, multistage, stratified probability sample of ED visits in the United States, administered by the National Center for Health Statistics, a branch of the Centers for Disease Control and Prevention. The NHAMCS-ED sample is collected from approximately 300 hospital-based EDs per year, randomly selected from approximately 1900 geographic areas in all 50 states. The survey uses a standardized data collection form to gather detailed information from approximately $100 \mathrm{pa}-$ tients per hospital-based ED.

\section{Study variables}

The primary outcome used for the study was the patients' depression status (whether the patients currently have depression identified by the variable "depression status" in the NHAMC-ED subfile). This status includes "affective disorders and major depressive disorders, such as episodes of depressive reaction, psychogenic depression, and reactive depression" (p. 148) [10].
Secondary outcomes included the Emergency Severity Index (ESI) score (a five-level ED triage algorithm assigning patients a score from 1 [most urgent] to 5 [least urgent] on the basis of acuity and resource needs), hospital admission, intensive care unit (ICU) admission, blood test, imaging (including X-ray, CT, ultrasound, and MRI), procedures (BiPAP/CPAP; bladder catheter; cast, splint, wrap; central line other; IV fluids; CPR; endotracheal intubation; incision \& drainage; IV fluids; lumbar puncture; nebulizer therapy; pelvic exam; skin adhesives; suturing/staples; other),whether the patient left before triage or treatment, length of stay, and whether the patient died in the ED/hospital.

Covariates included demographic characteristics (age, sex, race/ethnicity, region); socioeconomic status indicators, including residence (private home, nursing home, homeless, other) and insurance (private insurance, Medicare, Medicaid/CHIP, uninsured, other); day and mode of arrival; triage vital signs (temperature, pain scale, blood pressure, etc.); and reason for the ED visit. To assign a primary reason for each ED visit, we synthesized ten system-based symptom clusters from the nine symptom modules used in the NHAMCS (for reference, see p. 164 [10]). The detail of the coding algorithms and categories were introduced in the Appendicitis II of each years' survey file documentations. Generally, the reason for visit was coded into 8 main modules including symptom module, disease module, diagnostic module, screening and preventive module, treatment module, injuries and adverse effects module, test results module, and administrative module. In the current study, we used the symptom module, which were categorized into 10 groups including (1) general symptoms; (2) symptoms referable to psychological and mental disorders; (3) symptoms referable to the nervous system; (4) symptoms referable to the cardiovascular and lymphatic systems; (5) symptoms referable to the eyes and ears; (6) symptoms referable to the respiratory system; (7) symptoms referable to the digestive system; (8) symptoms referable to the genitourinary system; (9) symptoms referable to the skin, nails, and hair; (10) symptoms referable to the musculoskeletal system. Note that, as per the NHAMCS modules, our "Reason for ED Visit - Psychiatric" cluster excluded the following: alcoholism, adverse effects of alcohol, drug (prescription and illicit) addiction/dependence, drug intoxication, intentional drug overdose, and unintentional overdose.

\section{Statistical analyses}

Population characteristics between ED patients with versus those without depression were described and compared using $x^{2}$ tests. We used logistic regression to test for associations between the depression status and the covariates after adjusting for confounding factors. We 
also used logistic regression to investigate associations between depression and secondary outcomes, testing for mediation by covariates. The NHAMCS-ED dataset used in this analysis relies on a sequential hot-deck method to impute 3-digit ICD-9-CM codes (ICD-10-CM for 2016) for items such as age, sex, primary diagnosis, ED volume, and geographic region. Other variables were imputed with the median of the corresponding variables prior to generating the logistic regression models. We used SAS (Version 9.4) for our analysis, setting $\alpha=0.05$ as the statistical significance threshold. All odds ratios were calculated with $95 \%$ confidence intervals; $p$-values are $<0.05$ unless otherwise stated.

\section{Results}

In 2014-2016, there were 278,699,057 total adult ED visits in the US, corresponding to approximately 92,899 , 685 annual ED visits. Patients with depression made up approximately $31,878,551$ (11.4\%) (10,626,184 annually) of these visits. Basic characteristics are described in Table 1.

The proportion of ED visits by patients with depression varied by US census region: Northeast, $18.5 \%$; Midwest, 33.9\%; South, 31.5\%; and West, $16.1 \%(p<0.01)$. A greater proportion of ED patients with depression belonged to the $40-49,50-59$, and $60-74$ age groups as compared to their non-depressed counterparts (18.1 vs. $15.2 \%, 19.1$ vs. $14.8 \%$, and 17.8 vs. $15.3 \%$, respectively). ED patients with depression comprised a higher proportion of non-Hispanic Whites relative to those without depression (74.0 vs. $61.7 \%$ ).

Table 2 describes associations between ED patients' characteristics (demographic, socioeconomic, and clinical) and their depression status. Male ED patients were 38\% (aOR: 0.62; CI: 0.59-0.67) less likely than females to have depression. Among ED patients, Blacks were 38\% (aOR: 0.62; CI: 0.57-0.68) less likely than Whites to have depression; Hispanics, 49\% less likely (aOR:0.51; CI: 0.45-0.57); and Asians, 63\% less likely (aOR:0.37 CI: 0.27-0.51). Compared to ED patients inhabiting a private residence, those who were living in nursing homes or were homeless were $1.63(95 \%$ CI: $1.35-1.97)$ and 1.82 (CI: 1.46-2.26) times, respectively, more likely to have depression. Compared to ED patients with private insurance, those with Medicare and Medicaid or CHIP were 1.82 (95\% CI: $1.66-2.00$ ) and 1.74 times (CI: $1.60-1.89$ ), respectively, more likely to have depression. Compared to patients who arrived at the ED by other means, patients who arrived by ambulance were 1.25 times more likely (95\% CI: $1.16-1.36$ ) to have depression. ED patients who presented with overdose/poisoning were 2.19 times (CI: 1.75-2.74) more likely to have depression than those presenting with injury or trauma. ED patients who sought care for psychiatric reasons were 5.00 times
(95\% CI: 4.41-5.66) more likely to have depression than those seeking care for general symptoms (Table 3). Among ED patients with depression, males were 2.45 (95\% CI: $2.10-2.87$ ) times more likely than females to be seeking emergency care for psychiatric reasons and were 1.46 (95\% CI 1.03-2.05) times more likely than females to present with overdose/poisoning.

Tables 4 and 5 describe the proportions of ESI, hospital admission, ICU admission, and medical resources utilization, stratified by depression. In the weighted sample, $17.8 \%$ of the patients with depression were assigned immediate or emergent ESI score, which was higher than the patients with no depression (11.9\%). A total of $21.1 \%$ of the patients with depression were admitted into hospital after the ED visit, which was higher than the patients with no depression (12\%). A total of $2.6 \%$ of patients with depression were admitted into ICU, while the proportion for the patients with no depression was $1.6 \%$. Moreover, $59.8 \%$ of the patients with depression obtained a blood test, which is higher than the proportion for patients without depression (50.1\%). The hospital admission rate among ED patients was 1.50 times higher for patients with depression (CI: 1.38-1.63); depressed patients were also 1.33 times more likely to receive immediate vs. semi- or non-urgent ESI scores (CI: 1.181.49) compared to patients without depression. ED patients with depression were 1.33 (CI: 1.24-1.43) times more likely to receive blood tests but were less likely to access other resources medical resources; for example, they were $18 \%$ (CI: $0.59-1.14$ ) less likely to receive an MRI scan as compared to patients without depression.

\section{Discussion}

To our knowledge, this is the first, most comprehensive study describing the national characteristics of ED patients with depression. An insightful study by Ballou et al. [5] looked at US ED visits by depressed patients, but there are key differences between their work and ours. Namely, Ballou et al.'s scope was limited to ED visits for depressive complaints; further, they analyzed ED data from a different database (the Nationwide Emergency Department Sample), sampling 2 years (2006 and 2014) of data [5]. Studies on general ED use by patients with depression have been more limited than the present study in terms of sample size and national representativeness. For example, a prospective cohort study by Beiser et al. included a comparatively small sample $(n=999)$, as did Kumar et al.'s study of depression prevalence assessed in ED admissions $(n=536)[11,12]$. Beiser et al. and Kumar et al. reported markedly higher rates of depression (27 and 30\%, respectively) among ED patients than the rate noted here $(11.4 \%)[11,12]$. However, we must point out methodologic differences between their studies $[11,12]$ and ours. Whereas we used 
Table 1 Baseline Characteristics of Patients Presenting to the ED, Stratified by Depression, NHAMCS 2014-2016

\begin{tabular}{|c|c|c|c|c|c|c|}
\hline & \multicolumn{3}{|c|}{ Unweighted Sample (\%) } & \multicolumn{3}{|c|}{ Weighted Sample (\%) } \\
\hline & All & No Depression & Depression & All & No Depression & Depression \\
\hline & 42,832 & $37,737(88.1)$ & 5059 (11.9) & $278,699,057$ & $246,820,506(88.6)$ & $31,878,551(11.4)$ \\
\hline Male* & $18,469(43.1)$ & $16,649(44.1)$ & $1820(35.7)$ & $119,751,766(43.0)$ & $109,011,905(44.2)$ & $10,739,861(33.7)$ \\
\hline \multicolumn{7}{|l|}{ Age $^{*}$} \\
\hline $18-39$ & $17,912(41.8)$ & $16,085(42.6)$ & $1827(35.9)$ & $118,068,691(42.4)$ & $106,669,971(43.2)$ & $11,398,720(35.8)$ \\
\hline $40-49$ & $6662(15.6)$ & $5727(15.2)$ & $935(18.4)$ & $43,185,040(15.5)$ & $37,429,025(15.2)$ & $5,756,015(18.1)$ \\
\hline $50-59$ & $6707(15.7)$ & $5704(15.1)$ & $1003(19.7)$ & $42,679,091(15.3)$ & $36,593,175(14.8)$ & $6,085,916(19.1)$ \\
\hline $60-74$ & $6678(15.6)$ & $5807(15.4)$ & $871(17.1)$ & $43,420,164(15.6)$ & $37,739,318(15.3)$ & $5,680,845(17.8)$ \\
\hline$\geq 75$ & $4873(11.4)$ & $4414(11.7)$ & $459(9.0)$ & $31,346,071(11.2)$ & $28,389,016(11.5)$ & $2,957,055(9.3)$ \\
\hline \multicolumn{7}{|l|}{ Race/ethnicity* } \\
\hline White & $27,251(63.6)$ & $23,546(62.4)$ & $3705(72.7)$ & $175,775,546(63.1)$ & $152,171,847(61.7)$ & $23,603,698(74.0)$ \\
\hline Black & $9207(21.5)$ & $8299(22.0)$ & $908(17.8)$ & $62,663,628(22.5)$ & $57,104,372(23.1)$ & $5,559,256(17.4)$ \\
\hline Hispanic & $5152(12.0)$ & $4754(12.6)$ & $398(7.8)$ & $33,391,671(12.0)$ & $31,036,082(12.6)$ & $2,355,589(7.4)$ \\
\hline Asian & $804(1.9)$ & $760(2.0)$ & $44(0.9)$ & $4,392,213(1.6)$ & $4,221,497(1.7)$ & $170,717(0.5)$ \\
\hline Other & $418(1.0)$ & $378(1.0)$ & $40(0.8)$ & 2,475,999 (0.9) & 2,286,708 (0.9) & 189,291 (0.6) \\
\hline \multicolumn{7}{|l|}{ Residence type* } \\
\hline Private residence & $39,819(95.1)$ & $35,290(95.6)$ & 4529 (91.3) & $258,354,513(95.3)$ & $230,189,300(95.7)$ & $28,165,213(91.7)$ \\
\hline Nursing home & $885(2.1)$ & $703(1.9)$ & $182(3.7)$ & $5,875,161(2.2)$ & $4,597,878$ (1.9) & $1,277,283(4.2)$ \\
\hline Homeless & $534(1.3)$ & $392(1.1)$ & $142(2.9)$ & $2,480,109(0.9)$ & $1,835,949(0.8)$ & $644,160(2.1)$ \\
\hline Other & $651(1.6)$ & $541(1.5)$ & $110(2.2)$ & $4,501,686(1.7)$ & $3,885,684(1.6)$ & $616,002(2.0)$ \\
\hline \multicolumn{7}{|l|}{ Insurance type* } \\
\hline Private insurance & $12,446(30.8)$ & $11,293(31.7)$ & $1153(23.9)$ & $79,443,111(30.5)$ & $72,336,078(31.3)$ & $7,107,033(24.1)$ \\
\hline Medicare & $10,517(26.0)$ & 8972 (25.2) & $1545(32.1)$ & $66,956,323(25.7)$ & $57,073,540(24.7)$ & $9,882,783(33.5)$ \\
\hline Medicaid or CHIP & $11,148(27.6)$ & 9467 (26.6) & $1681(34.9)$ & $71,529,605(27.5)$ & $61,857,136(26.8)$ & $9,672,469(32.8)$ \\
\hline Uninsured & $4886(12.1)$ & $4564(12.8)$ & $322(6.7)$ & $33,248,283(12.8)$ & $31,145,408(13.5)$ & $2,102,876(7.1)$ \\
\hline Other & $1406(3.5)$ & $1289(3.6)$ & $117(2.4)$ & $9,371,908$ (3.6) & $8,660,813(3.7)$ & 711,095 (2.4) \\
\hline \multicolumn{7}{|l|}{ Year* } \\
\hline 2014 & $15,319(35.8)$ & $13,677(36.2)$ & $1642(32.2)$ & $90,554,699(32.5)$ & $81,304,754(32.9)$ & $9,249,945(29.0)$ \\
\hline 2015 & $14,041(32.8)$ & $12,364(32.8)$ & 1677 (32.9) & $89,005,064(31.9)$ & $78,981,947(32.0)$ & $10,023,117(31.4)$ \\
\hline 2016 & $13,472(31.5)$ & $11,696(31.0)$ & 1776 (34.9) & $99,139,294(35.6)$ & $86,533,805(35.1)$ & $12,605,489(39.5)$ \\
\hline \multicolumn{7}{|l|}{ Day of ED Visit } \\
\hline Sunday & $5622(13.1)$ & $4964(13.2)$ & $658(12.9)$ & $35,918,011(12.9)$ & $31,810,077(12.9)$ & $4,107,933(12.9)$ \\
\hline Monday & $6930(16.2)$ & $6146(16.3)$ & $784(15.4)$ & $44,958,717(16.1)$ & $39,878,806(16.2)$ & $5,079,911(15.9)$ \\
\hline Tuesday & $6347(14.8)$ & $5614(14.9)$ & $733(14.4)$ & $40,922,676(14.7)$ & $36,423,291(14.8)$ & 4,499,385 (14.1) \\
\hline Wednesday & $6225(14.5)$ & $5489(14.5)$ & $736(14.4)$ & $40,888,226(14.7)$ & $36,088,222(14.6)$ & $4,800,003(15.1)$ \\
\hline Thursday & $5952(13.9)$ & $5211(13.8)$ & $741(14.5)$ & $39,069,043(14.0)$ & $34,301,499(13.9)$ & $4,767,544(15.0)$ \\
\hline Friday & $5960(13.9)$ & $5253(13.9)$ & 707 (13.9) & $38,869,467(13.9)$ & $34,729,896(14.1)$ & $4,139,571(13.0)$ \\
\hline Saturday & $5796(13.5)$ & $5060(13.4)$ & $736(14.4)$ & $38,072,918(13.7)$ & $33,588,715(13.6)$ & $4,484,203(14.1)$ \\
\hline Arrive by ambulance* & $7729(18.5)$ & $6462(17.6)$ & $1267(25.4)$ & $49,769,047(18.3)$ & $41,864,145(17.4)$ & $7,904,902(25.3)$ \\
\hline Seen within last $72 \mathrm{~h}$ & $1914(4.9)$ & $1668(4.9)$ & $246(5.3)$ & $11,953,039(4.8)$ & $10,393,280(4.7)$ & $1,559,759(5.5)$ \\
\hline \multicolumn{7}{|c|}{ Pain level at Presentation } \\
\hline No pain & $7711(24.4)$ & $6654(23.8)$ & $1057(28.5)$ & $46,478,004(23.1)$ & $40,809,768(22.9)$ & $5,668,235(25.2)$ \\
\hline Mild & $2916(9.2)$ & $2667(9.5)$ & $249(6.7)$ & $18,235,636(9.1)$ & $16,637,092(9.3)$ & $1,598,544(7.1)$ \\
\hline Moderate & $9430(29.8)$ & 8429 (30.2) & $1001(27.0)$ & $60,509,861(30.1)$ & $54,315,526(30.4)$ & $6,194,335$ (27.6) \\
\hline
\end{tabular}


Table 1 Baseline Characteristics of Patients Presenting to the ED, Stratified by Depression, NHAMCS 2014-2016 (Continued)

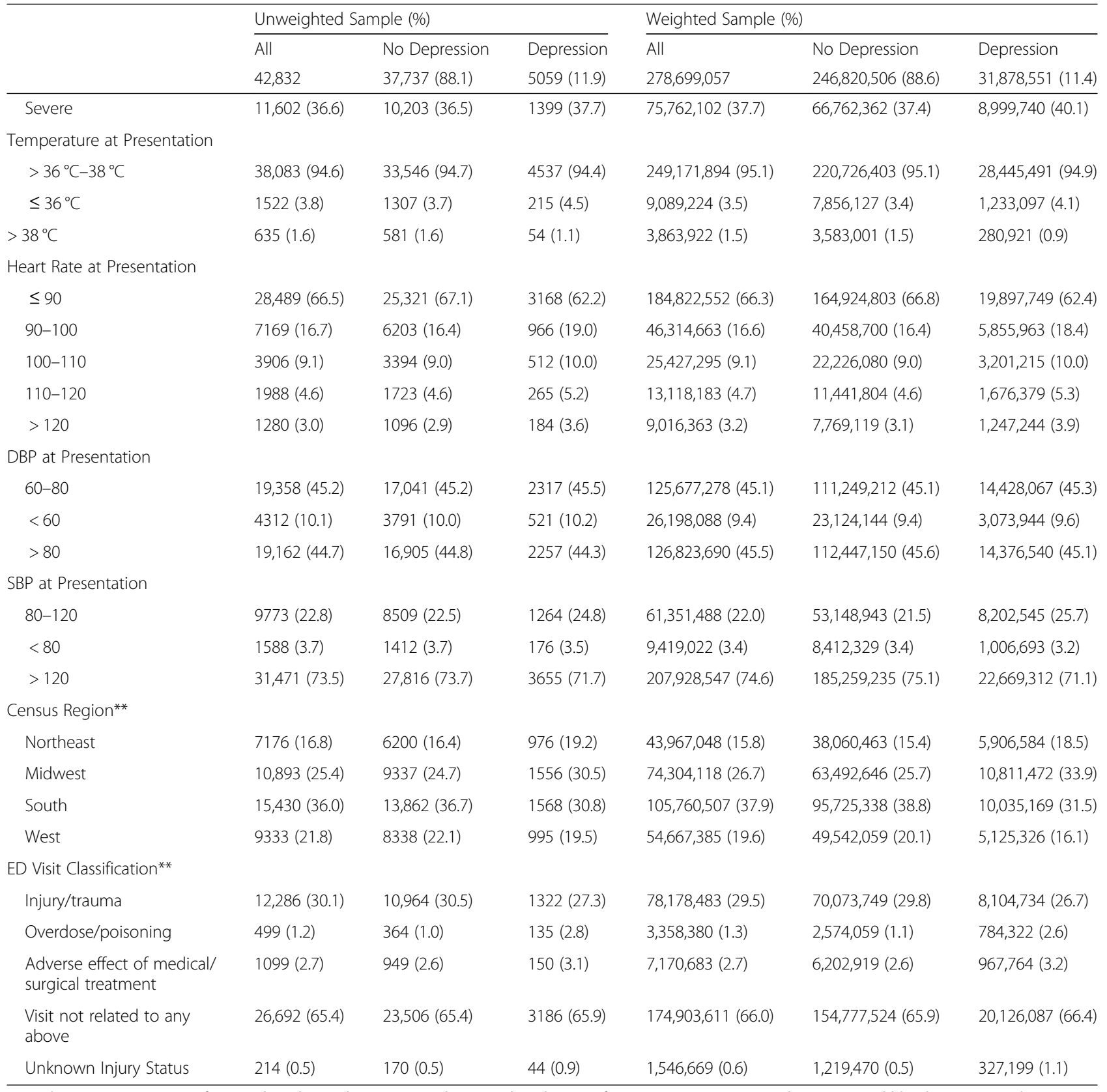

Note: the missing proportion for arrival mode, residency type, and injury is less than 5\%; for insurance, temperature, heart rate, and blood pressure, and seen within last 72 h, 5-10\%; for triage level, $27 \%$; for pain level, $26 \%$

${ }^{*} p<0.05,{ }^{* *} p<0.01$

NHAMCS data on ED patients' depression status, these studies $[11,12]$ relied on self-reported depression questionnaires administered to patients in their ED. Further, our study characterizes ED patients with depression with greater power by using 3 years of data from a larger, more representative sample. Our study also provides previously unreported data concerning vitals and other clinical information in ED patients with depression.

From 2014 to 2016, patients with depression made more than 10 million ED visits annually. Compared to patients without depression, those with depression had higher rates of hospital admission and ICU admission. We did not observe associations between ED patients' depression and any somatic reasons for ED visit. This finding suggests that comorbid conditions that have previously been correlated with depression (e.g., rheumatoid arthritis) may not strongly predict these patients' emergency care needs [12]. However, patients with depression were more likely to seek emergency treatment for psychiatric symptoms and for acute overdose/poisoning. 
Table 2 Association Between Depression Status in ED Patients and Their Visiting Characteristics (NHAMCS 2014-2016)

\begin{tabular}{|c|c|c|}
\hline & Crude OR (95\% CI) & Adjusted OR (95\% Cl) \\
\hline \multicolumn{3}{|l|}{ Age } \\
\hline $18-39$ & Reference [1] & Reference [1] \\
\hline $40-49$ & $1.44(1.32-1.56)$ & $1.41(1.29-1.55)$ \\
\hline $50-59$ & $1.55(1.43-1.68)$ & $1.48(1.35-1.62)$ \\
\hline $60-74$ & $1.32(1.21-1.44)$ & $1.03(0.93-1.14)$ \\
\hline$\geq 75$ & $0.92(0.82-1.02)$ & $0.52(0.45-0.60)$ \\
\hline Male vs. Female & $0.70(0.66-0.75)$ & $0.62(0.59-0.67)$ \\
\hline \multicolumn{3}{|l|}{ Race/ethnicity } \\
\hline White & Reference [1] & Reference [1] \\
\hline Black & $0.70(0.64-0.75)$ & $0.62(0.57-0.68)$ \\
\hline Hispanic & $0.53(0.48-0.59)$ & $0.51(0.45-0.57)$ \\
\hline Asian & $0.37(0.27-0.50)$ & $0.37(0.27-0.51)$ \\
\hline Other & $0.67(0.49-0.93)$ & $0.65(0.46-0.91)$ \\
\hline \multicolumn{3}{|l|}{ Day of Week } \\
\hline Sunday & Reference [1] & Reference [1] \\
\hline Monday & $0.96(0.86-1.07)$ & $0.95(0.85-1.07)$ \\
\hline Tuesday & $0.99(0.88-1.10)$ & $0.94(0.84-1.06)$ \\
\hline Wednesday & $1.01(0.90-1.13)$ & $0.99(0.88-1.12)$ \\
\hline Thursday & $1.07(0.96-1.20)$ & $1.06(0.94-1.19)$ \\
\hline Friday & $1.02(0.91-1.14)$ & $0.97(0.86-1.09)$ \\
\hline Saturday & $1.10(0.98-1.23)$ & $1.07(0.95-1.21)$ \\
\hline \multicolumn{3}{|l|}{ Year } \\
\hline 2014 & Reference [1] & Reference [1] \\
\hline 2015 & $1.13(1.05-1.21)$ & $1.09(1.01-1.18)$ \\
\hline 2016 & $1.27(1.18-1.36)$ & $1.22(1.13-1.32)$ \\
\hline \multicolumn{3}{|l|}{ Residence type } \\
\hline Private residence & Reference [1] & Reference [1] \\
\hline Nursing home & $2.01(1.70-2.37)$ & $1.63(1.35-1.97)$ \\
\hline Homeless & $2.81(2.31-3.41)$ & $1.82(1.46-2.26)$ \\
\hline Other & $1.58(1.28-1.94)$ & $1.29(1.03-1.61)$ \\
\hline \multicolumn{3}{|l|}{ Insurance type } \\
\hline Private insurance & Reference [1] & Reference [1] \\
\hline Medicare & $1.60(1.48-1.74)$ & $1.82(1.66-2.00)$ \\
\hline Medicaid or CHIP & $1.74(1.61-1.88)$ & 1.74(1.60-1.89) \\
\hline Uninsured & $0.69(0.61-0.79)$ & $0.76(0.66-0.87)$ \\
\hline Other & $0.89(0.73-1.08)$ & $1.03(0.84-1.26)$ \\
\hline \multicolumn{3}{|l|}{ Temperature } \\
\hline $36^{\circ} \mathrm{C}-38^{\circ} \mathrm{C}$ & Reference [1] & Reference [1] \\
\hline$\leq 36^{\circ} \mathrm{C}$ & $1.22(1.06-1.42)$ & $1.17(1.00-1.36)$ \\
\hline$>38^{\circ} \mathrm{C}$ & $0.69(0.52-0.92)$ & $0.65(0.49-0.87)$ \\
\hline \multicolumn{3}{|l|}{ Heart Rate } \\
\hline$\leq 90$ & Reference [1] & Reference [1] \\
\hline $90-100$ & $1.25(1.15-1.35)$ & $1.20(1.10-1.30)$ \\
\hline $100-110$ & $1.21(1.09-1.33)$ & $1.11(1.00-1.24)$ \\
\hline
\end{tabular}


Table 2 Association Between Depression Status in ED Patients and Their Visiting Characteristics (NHAMCS 2014-2016) (Continued)

\begin{tabular}{|c|c|c|}
\hline & Crude OR (95\% Cl) & Adjusted OR $(95 \% \mathrm{Cl})$ \\
\hline $110-120$ & $1.23(1.08-1.41)$ & $1.13(0.98-1.30)$ \\
\hline$>120$ & $1.34(1.14-1.58)$ & $1.21(1.02-1.44)$ \\
\hline \multicolumn{3}{|l|}{ DBP } \\
\hline $60-80$ & Reference [1] & Reference [1] \\
\hline$<60$ & $1.01(0.91-1.12)$ & $0.98(0.88-1.09)$ \\
\hline$>80$ & $0.98(0.92-1.05)$ & $0.94(0.88-1.01)$ \\
\hline \multicolumn{3}{|l|}{ Pain level } \\
\hline No pain & Reference [1] & Reference [1] \\
\hline Mild & $0.59(0.51-0.68)$ & $0.77(0.66-0.90)$ \\
\hline Moderate & $0.83(0.76-0.89)$ & $0.98(0.90-1.07)$ \\
\hline Severe & $0.86(0.79-0.94)$ & $1.10(1.00-1.22)$ \\
\hline 72-h revisit vs. not & $0.91(0.80-1.05)$ & $0.90(0.78-1.04)$ \\
\hline Arrival by Ambulance vs. Not & $1.60(1.50-1.72)$ & $1.25(1.16-1.36)$ \\
\hline \multicolumn{3}{|l|}{ Census Region } \\
\hline Northeast & Reference [1] & Reference [1] \\
\hline Midwest & $1.06(0.97-1.15)$ & 1.08(0.99-1.19) \\
\hline South & $0.72(0.66-0.78)$ & $0.81(0.74-0.89)$ \\
\hline West & $0.76(0.69-0.83)$ & $0.79(0.72-0.88)$ \\
\hline \multicolumn{3}{|l|}{ Reason for Visit (by Symptom Module) } \\
\hline General & Reference [1] & Reference [1] \\
\hline Psychiatric & $5.03(4.48-5.65)$ & $5.00(4.41-5.66)$ \\
\hline Neurologic & $0.87(0.76-0.98)$ & $0.82(0.72-0.93)$ \\
\hline Cardiovascular and Lymphatic & $0.66(0.52-0.83)$ & $0.68(0.54-0.87)$ \\
\hline Eyes and/or Ears & $0.55(0.42-0.71)$ & $0.59(0.45-0.77)$ \\
\hline Respiratory & $0.78(0.69-0.88)$ & $0.77(0.68-0.87)$ \\
\hline Digestive & $0.79(0.71-0.87)$ & $0.78(0.70-0.87)$ \\
\hline Genitourinary & $0.58(0.49-0.68)$ & $0.60(0.50-0.70)$ \\
\hline Dermatologic & $0.56(0.45-0.69)$ & $0.60(0.48-0.75)$ \\
\hline Musculoskeletal & $0.72(0.65-0.80)$ & $0.75(0.67-0.84)$ \\
\hline Other & $0.82(0.74-0.90)$ & $0.88(0.78-0.99)$ \\
\hline \multicolumn{3}{|l|}{ Reason for Visit (by Injury Type) } \\
\hline Injury/Trauma & Reference [1] & Reference [1] \\
\hline Overdose/Poisoning & $3.08(2.51-3.78)$ & $2.19(1.75-2.74)$ \\
\hline Adverse effect of medical/surgical treatment & $1.31(1.09-1.57)$ & $1.23(1.01-1.48)$ \\
\hline Visit not related to any above & $1.13(1.06-1.21)$ & $1.12(1.03-1.22)$ \\
\hline Questionable injury status & $2.15(1.53-3.01)$ & $1.45(1.01-2.09)$ \\
\hline
\end{tabular}

Note: the adjusted OR was from a logistic regression including all variables in the table

Demographic factors were associated with the prevalence of depression in ED patients. In terms of region, EDs in the Midwest had the greatest proportion of visits by patients with depression. Female ED patients were more likely than males to have depression, as were nonHispanic Whites compared to other races/ethnicities, particularly Asians. These gender and racial/ethnic differences are roughly concordant with US demographic patterns in depression prevalence observed beyond the ED setting [13]. However, these patterns have been problematized by research indicating that certain nonWhite minority populations are less likely to receive or seek mental health diagnoses and care [14-16]. If the large proportion of Whites among patients with depression partly reflects such differences in diagnosis and treatment seeking, there may in fact be a number of 
Table 3 Selected Reason for Visit and Emergency Department Diagnosis among ED Patients with Depression, NHAMCS 2014-2016

\begin{tabular}{|c|c|c|c|c|c|c|}
\hline \multirow[t]{2}{*}{ Reason for ED Visit } & \multicolumn{3}{|c|}{ Unweighted Sample (\%) } & \multicolumn{3}{|c|}{ Weighted Sample (\%) } \\
\hline & All & No Depression & Depression & All & No Depression & Depression \\
\hline General & $8187(19.1)$ & $7126(18.9)$ & $1061(20.8)$ & $53,664,580(19.3)$ & $46,862,329(19.0)$ & $6,802,251(21.4)$ \\
\hline Psychiatric & $1700(4.0)$ & $972(2.6)$ & $728(14.3)$ & $9,426,523(3.4)$ & $5,550,218(2.3)$ & $3,876,305(12.2)$ \\
\hline Neurologic & $3304(7.7)$ & $2927(7.8)$ & $377(7.4)$ & $20,833,741(7.5)$ & $18,542,430(7.5)$ & $2,291,311(7.2)$ \\
\hline Cardiovascular and Lymphatic & $889(2.1)$ & $810(2.2)$ & $79(1.6)$ & $5,993,917(2.2)$ & $5,512,635(2.2)$ & $481,282(1.5)$ \\
\hline Eyes and/or Ears & $848(2.0)$ & $784(2.1)$ & $64(1.3)$ & $5,778,778(2.1)$ & $5,233,633(2.1)$ & $545,145(1.7)$ \\
\hline Respiratory & $4198(9.8)$ & $3762(10.0)$ & $436(8.6)$ & $27,856,021(10.0)$ & $25,021,474(10.2)$ & $2,834,547(8.9)$ \\
\hline Digestive & $6807(15.9)$ & $6093(16.2)$ & $714(14.0)$ & $46,038,272(16.5)$ & $41,187,597(16.7)$ & $4,850,675(15.2)$ \\
\hline Genitourinary & $2477(5.8)$ & $2281(6.1)$ & $196(3.9)$ & $14,984,361(5.4)$ & $13,934,779(5.7)$ & $1,049,581(3.3)$ \\
\hline Dermatologic & $1333(3.1)$ & $1231(3.3)$ & $102(2.0)$ & $8,716,118(3.1)$ & $8,018,952(3.3)$ & $697,166(2.2)$ \\
\hline Musculoskeletal & 6519 (15.2) & 5891 (15.6) & $628(12.3)$ & $42,820,579(15.4)$ & $38,855,925(15.8)$ & $3,964,654(12.5)$ \\
\hline Other & $6501(15.2)$ & $5796(15.4)$ & 705 (13.9) & $42,147,135$ & $37,695,947(15.3)$ & $4,451,188(14.0)$ \\
\hline
\end{tabular}

non-White patients with unaddressed mental health care needs in the ED.

Notably, patients with depression who sought ED treatment for psychiatric symptoms were roughly two times more likely to be male than female, consistent with findings by Ballou et al. [5]. Because our psychiatric
ED visit classification excluded visits related to alcoholism and other substance use disorders, our finding of higher rates of psychiatric ED visits for depressed male patients cannot be well explained by males' higher rates of alcoholism and substance use disorders in the general population [3]. Given the social barriers that men,

Table 4 Proportion of Emergency Severity Index, Hospital admission, ICU admission, Medical resources utilization, stratified by Depression, NHAMCS 2014-2016

\begin{tabular}{|c|c|c|c|c|c|c|}
\hline & \multicolumn{3}{|l|}{ Unweighted Sample } & \multicolumn{3}{|l|}{ Weighted Sample } \\
\hline & $\overline{\text { All }}$ & No Depression & Depression & $\overline{\text { All }}$ & No Depression & Depression \\
\hline \multicolumn{7}{|l|}{ ESI score s** $^{* *}$} \\
\hline 1 (Immediate) & $239(0.8)$ & $210(0.8)$ & $29(0.8)$ & $1,496,327(0.8)$ & $1,272,228(0.7)$ & $224,099(1.0)$ \\
\hline 2 (Emergent) & $3615(11.6)$ & 2966 (10.9) & $649(16.9)$ & $23,433,327(11.8)$ & $19,516,968(11.2)$ & $3,916,359(16.8)$ \\
\hline 3 (Urgent) & $15,392(49.5)$ & $13,428(49.3)$ & $1964(51.0)$ & $97,000,149(49.0)$ & $85,337,086(48.8)$ & $11,663,063(50.1)$ \\
\hline 4 (Semi-urgent) & $10,051(32.3)$ & 9037 (33.2) & $1014(26.3)$ & $65,085,335(32.9)$ & $58,899,226(33.7)$ & $6,186,109(26.6)$ \\
\hline 5 (Non-urgent) & $1784(5.7)$ & $1589(5.8)$ & $195(5.1)$ & $11,046,598(5.6)$ & $9,768,544(5.6)$ & $1,278,054(5.5)$ \\
\hline Hospital Admission & $5852(13.7)$ & $4764(12.6)$ & $1088(21.4)$ & $36,388,538(13.1)$ & $29,653,166(12.0)$ & $6,735,373(21.1)$ \\
\hline $\mathrm{ICU}^{*}$ & $698(1.6)$ & $587(1.6)$ & $111(2.2)$ & $4,647,353(1.7)$ & $3,833,519(1.6)$ & $813,835(2.6)$ \\
\hline Death in ED or hospital & $201(0.5)$ & $187(0.5)$ & $14(0.3)$ & $1,342,510(0.5)$ & $1,224,939(0.5)$ & $117,571(0.4)$ \\
\hline Left before/after triage* & $1085(2.5)$ & $978(2.6)$ & $107(2.1)$ & $6,792,175(2.4)$ & $6,134,329(2.5)$ & $657,846(2.1)$ \\
\hline Blood test performed* & $21,958(51.3)$ & $18,860(50.0)$ & $3098(60.8)$ & $142,656,097(51.2)$ & $123,598,652(50.1)$ & $19,057,445(59.8)$ \\
\hline Any imaging performed & $21,950(51.2)$ & $19,494(51.7)$ & $2456(48.2)$ & $144,824,612(52.0)$ & $128,979,436(52.3)$ & $15,845,177(49.7)$ \\
\hline $\mathrm{X}$-ray in $\mathrm{ED}$ & 15,099 (35.3) & $13,363(35.4)$ & $1736(34.1)$ & $99,429,274(35.7)$ & $88,271,718(35.8)$ & $11,157,556(35.0)$ \\
\hline$C T$ in $E D$ & $8414(19.6)$ & 7392 (19.6) & $1022(20.1)$ & $54,986,804(19.7)$ & $48,528,197(19.7)$ & $6,458,608(20.3)$ \\
\hline Ultrasound in ED & $2218(5.2)$ & $2012(5.3)$ & $206(4.0)$ & $14,936,538(5.4)$ & $13,580,831(5.5)$ & $1,355,707(4.3)$ \\
\hline $\mathrm{MRI}$ in $\mathrm{ED}^{*}$ & $446(1.0)$ & $402(1.1)$ & $44(0.9)$ & $2,831,626(1.0)$ & $2,594,364(1.1)$ & $237,262(0.7)$ \\
\hline Other Imaging in ED & $604(1.4)$ & $539(1.4)$ & $65(1.3)$ & $4,297,097(1.5)$ & $3,896,319(1.6)$ & $400,778(1.3)$ \\
\hline Procedure & $21,021(49.1)$ & $18,626(49.4)$ & $2395(47.0)$ & $133,801,012(48.0)$ & $119,211,149$ (48.3) & $14,589,863(45.8)$ \\
\hline $\begin{array}{l}\text { Waiting time (min, } \\
\text { means }[95 \% \mathrm{Cl}] \text { ) }\end{array}$ & $41.1(40.3-41.8)$ & $41.0(40.2-41.8)$ & $41.7(39.3-44.1)$ & $39.9(39.2-40.6)$ & $39.8(39.0-40.5)$ & $41.0(38.9-43.2)$ \\
\hline $\begin{array}{l}\text { Visit length** (min, } \\
\text { means }[95 \% \mathrm{CI}])\end{array}$ & $245.6(241.6-249.6)$ & $237.5(233.4-241.6)$ & 309.1 (293.5-324.6) & $230.2(226.7-233.8)$ & $224.8(221.2-228.5)$ & $275.2(262.4-288.0)$ \\
\hline
\end{tabular}


Table 5 Odds Ratio of Emergency Severity Index, Hospital Admission, ICU Admission, Medical Resources Utilization for Patients with versus without Depression, NHAMCS 2014-2016

\begin{tabular}{|c|c|c|c|c|}
\hline & Crude Odds & Adjusted for vari & & \\
\hline & & Demographic & + Socioeconomic & + Visiting \& Clinical \\
\hline ESI Score: 1 or 2 vs. 4 or 5 & $1.88(1.69-2.08)$ & $1.93(1.74-2.14)$ & $1.85(1.67-2.06)$ & $1.33(1.18-1.49)$ \\
\hline ESI Score: Urgent vs. Semi- or Non-Urgent & $1.29(1.19-1.39)$ & $1.28(1.18-1.38)$ & $1.25(1.16-1.35)$ & $1.11(1.02-1.20)$ \\
\hline Hospital Admission & $1.88(1.75-2.02)$ & $2.01(1.86-2.17)$ & 1.84(1.70-1.99) & $1.50(1.38-1.63)$ \\
\hline ICU & $1.41(1.15-1.73)$ & 1.46(1.19-1.80) & $1.32(1.07-1.63)$ & $1.10(0.88-1.37)$ \\
\hline Death & $0.55(0.32-0.95)$ & $0.62(0.36-1.07)$ & $0.58(0.33-1.00)$ & $0.43(0.24-0.77)$ \\
\hline Left & $0.81(0.66-0.99)$ & $0.81(0.66-0.99)$ & $0.80(0.65-0.98)$ & $0.77(0.62-0.95)$ \\
\hline Blood test & $1.55(1.46-1.65)$ & $1.54(1.45-1.63)$ & $1.52(1.43-1.61)$ & $1.33(1.24-1.43)$ \\
\hline Any imaging & $0.87(0.82-0.92)$ & $0.85(0.80-0.90)$ & $0.84(0.79-0.90)$ & $0.89(0.84-0.95)$ \\
\hline X-ray & $0.94(0.89-1.00)$ & $0.93(0.87-0.99)$ & $0.92(0.87-0.99)$ & $0.95(0.89-1.02)$ \\
\hline $\mathrm{CT}$ & $1.03(0.96-1.11)$ & $1.02(0.95-1.10)$ & $1.01(0.94-1.09)$ & $0.97(0.89-1.05)$ \\
\hline Ultrasound & $0.75(0.65-0.87)$ & $0.73(0.63-0.84)$ & $0.74(0.64-0.86)$ & $0.84(0.71-0.98)$ \\
\hline MRI & $0.81(0.59-1.11)$ & $0.79(0.58-1.08)$ & $0.84(0.61-1.16)$ & $0.82(0.59-1.14)$ \\
\hline Procedure & $0.90(0.85-0.96)$ & $0.90(0.85-0.96)$ & $0.90(0.85-0.96)$ & $0.92(0.86-0.97)$ \\
\hline
\end{tabular}

a"Demographic" includes gender, age group, and race/ethnicity; "socioeconomic" includes residence type, insurance type, and census region; "visiting \& clinical" includes year, day of the week, arrival by ambulance, seen within last $72 \mathrm{~h}$, pain level, temperature, heart rate, dialytic blood pressure, injury status, and reason for visit

relative to women, face in seeking mental health care, this disparity may indicate a need for more routine depression care and/or screening in men to reduce their rate of psychiatric emergencies [17, 18]. Further, in Ballou et al.'s study, men were modestly more likely to present to the ED with self-harm [5]. In our sample, males with depression were more likely to present with overdose/poisoning-a finding that does not necessarily point to increased self-harm but bears highlighting for future inquiry. Additional data are needed to clarify the reasons for and extent of these gender-based disparities.

Compared to their non-depressed counterparts, patients with depression in the ED have higher ESI scores, hospital admission, and ICU admission, indicating that patients with depression require a higher level of emergency care. With regard to these outcomes, it is worth noting similarities between ED patients with depression and ED patients with cancer, whose utilization is higher across many dimensions of care [7, 19]. ED patients with cancer were also had higher odds of having depression [20]. The factors contributing to these outcomes in both patient populations may be the subject of future research. Understanding the reasons for ED revisits among patients with depression may facilitate the development of interventions or guidelines to reduce ED visit and revisit rates. Considering the substantial number of patients with depression in the ED, we suggest that the ED is an understudied setting for depression treatment. Finer-grained data on patients with a history of depression may inform ways of increasing this population's use of routine care over emergency care options.

\section{Limitations}

In the patient histories documented in the NHAMCSED data, patients are coded as either having or not having "depression status." Information including depression severity, subtype, and duration were not specified in this dataset. Such information may have predictive value; for example, in Beiser et al.'s prospective cohort study, a $10 \%$ increase in depression severity was correlated with a $10 \%$ increase in future ED visits [11]. Further, apart from depression, the NHAMCS-ED dataset does not indicate other mental disorders in the ED patient sample. We therefore could not adjust for potential psychiatric comorbidities. More generally, other comorbidities that are not documented in the dataset, such as chronic pain disorders, could play a role in mediating some of the associations we observed [21]. Another limitation of the NHAMCS-ED dataset is a lack of information on treatment history (pharmacologic or otherwise) for depression. Future studies may examine ED patients' specific depression characteristics, comorbidities, treatment history, and other finer-grained clinical data allowing for more refined associative and predictive models.

\section{Conclusions}

Our study advances understanding of the characteristics and clinical presentation of patients with depression in the ED. It is an initial step toward improving their care and clinical outcomes and reducing this population's ED burden. The study revealed the characteristics of ED patients with depression in a diverse, national sample. Patients with depression make more than 10 million ED 
visits annually. We found significant gender, age, racial/ ethnic, and regional differences among these patients. In the ED, patients with depression have significantly higher hospital and ICU admission rates compared to those without depression, indicating that patients with depression may require a higher level of emergency care. These findings argue for increasing recognition of the potential of the ED as a high-leverage setting for improving treatment and screening of depression, by identifying characteristics and trajectories of patients presenting to the ED with depression.

\section{Abbreviations \\ aOR: Adjusted Odds Ratio; Cl: Confidential Interval; ED: Emergency Department; NHAMCS-ED: National Hospital Ambulatory Medical Care Survey ED Subfile; ESI: Emergency Severity Index; ICU: Intensive care unit; CT: Computed tomography; MRI: Magnetic Resonance Imaging}

\section{Acknowledgements}

None.

\section{Conflict of interest disclosures}

None reported.

\section{Authors' contributions}

XZ had full access to all the data in the study and take responsibility for the integrity of the data and the accuracy of the data analysis. Concept and design: TH, XZ. Acquisition, analysis, or interpretation of data: All authors. Drafting of the manuscript: TH. Critical revision of the manuscript for important intellectual content: YJ, CRF, LAD, CKB. Statistical analysis: XZ. Obtained funding: XZ. Administrative, technical, or material support: TH, XZ. All authors have read and approved the manuscript.

\section{Funding}

This study was supported by Michigan Institute for Clinical and Health Research (MICHR No. UL1TR002240). The funders played no role in the design of the study, collection, analysis or interpretation of data, or writing of the manuscript.

\section{Availability of data and materials}

The NHAMCS-ED dataset can be accessed through the website of the US Centers for Disease Control and Prevention (CDC) (https://www.cdc.gov/ nchs/ahcd/index.htm). The detailed explanation of the survey data for each year and the code book can be found here: https://ftp.cdc.gov/pub/Health_ Statistics/NCHS/dataset_documentation/nhamcs/.

\section{Ethics approval and consent to participate}

This study was a secondary analysis of a public database and did not require ethical approval.

\section{Consent for publication}

Not applicable.

\section{Competing interests}

The authors declare that they have no competing interests. The funders/ sponsors had no role in the design and conduct of the study; collection, management, analysis, and interpretation of the data; preparation, review, or approval of the manuscript; and decision to submit the manuscript for publication

\section{Author details}

'Department of Psychology, University of Michigan College of Literature, Science, and the Arts, Ann Arbor, MI, USA. ${ }^{2}$ Department of Systems, Populations, and Leadership, University of Michigan School of Nursing, Room 1177, 400 North Ingalls Building, Ann Arbor, Ml 48109-5482, USA

${ }^{3}$ Department of Health Behavior and Biological Sciences, University of Michigan School of Nursing, Ann Arbor, MI, USA. ${ }^{4}$ Department of Emergency Medicine, University of Michigan School of Medicine, Ann Arbor, MI, USA.
${ }^{5}$ Department of Psychiatry, University of Michigan School of Medicine, Ann Arbor, MI, USA.

Received: 28 February 2020 Accepted: 16 June 2020

Published online: 22 June 2020

\section{References}

1. Lépine JP, Briley M. The increasing burden of depression. Neuropsychiatr Dis Treat. 2011;7(SUPPL):3-7.

2. Greenberg PE, Fournier AA, Sisitsky T, Pike CT, Kessler RC. The economic burden of adults with major depressive disorder in the United States (2005 and 2010). J Clin Psychiatry. 2015;76:155-62.

3. Substance Abuse and Mental Health Services Administration. Key substance use and mental health indicators in the United States: Results from the 2017 National Survey on Drug Use and Health. Accessed 12 July 2019, at http://www.samhsa.gov/data/.

4. Hazlett SB, McCarthy ML, Londner MS, Onyike CU. Epidemiology of adult psychiatric visits to U.S. emergency departments. Acad Emerg Med. 2004;11: 193-5.

5. Ballou S, Mitsuhashi S, Sankin LS, Petersen TS, Zubiago J, Lembo C, et al. Emergency department visits for depression in the United States from 2006 to 2014. Gen Hosp Psychiatry. 2019;59(April):14-9. https://doi.org/10.1016/j. genhosppsych.2019.04.015.

6. Hackman A, Goldberg R, Brown C, Fang L, Dickerson F, Wohlheiter K, et al. Use of emergency department services for somatic reasons by people with serious mental illness. Psychiatr Serv. 2006;57:563-6. https://doi.org/10.1176/ appi.ps.57.4.563.

7. Rivera DR, Gallicchio L, Brown J, Liu B, Kyriacou DN, Shelburne N. Trends in adult cancer-related emergency department utilization: an analysis of data from the nationwide emergency department sample. JAMA Oncol. 2017;3: e172450.

8. Asao K, Kaminski J, McEwen LN, Wu X, Lee JM, Herman WH. Assessing the burden of diabetes mellitus in emergency departments in the United States: the National Hospital Ambulatory Medical Care Survey (NHAMCS). J Diabetes Complicat. 2014;28:639-45. https://doi.org/10.1016/j.jdiacomp.2014. 02.005 .

9. National Center for Health Statistics, Centers for Disease Control and Prevention. National Hospital Ambulatory Medical Care Survey: 2016 NHAMCS micro-data file documentation. Accessed 12 July 2019, at https:// www.cdc.gov.

10. National Center for Health Statistics, Centers for Disease Control and Prevention. National Ambulatory Medical Care Survey: 2015 NHAMCS microdata file documentation. Accessed 12 July 2019, at https://www.cdc.gov.

11. Beiser DG, Ward CE, Vu M, Laiteerapong N, Gibbons RD. Depression in Emergency Department Patients and Association With Health Care Utilization. Acad Emerg Med. 2019;26(8):878-88. https://doi.org/10.1111/ acem.13726

12. Kumar A, Clark S, Boudreaux ED, Camargo CA. A multicenter study of depression among emergency department patients. Acad Emerg Med. 2004:11:1284-9

13. Olfson M, Blanco C, Marcus SC. Treatment of adult depression in the United States. JAMA Intern Med. 2016;176:1482-91.

14. Alegría M, Chatterji P, Wells $K$, Cao Z, Chen C, Takeuchi D, et al. Disparity in depression treatment among racial and ethnic minority populations in the United States. Psychiatr Serv. 2015:59:1264-72.

15. Zuvekas SH, Fleishman JA. Self-rated mental health and racial/ethnic disparities in mental health service use. Med Care. 2008;46:915-23.

16. Hines AL, Cooper LA, Shi L. Racial and ethnic differences in mental healthcare utilization consistent with potentially effective care: the role of patient preferences. Gen Hosp Psychiatry. 2017;46:14-9. https://doi.org/10. 1016/.j.genhosppsych.2017.02.002

17. Seidler ZE, Dawes AJ, Rice SM, Oliffe JL, Dhillon HM. The role of masculinity in men's help-seeking for depression: a systematic review. Clin Psychol Rev. 2016;49:106-18. https://doi.org/10.1016/j.cpr.2016.09.002.

18. M-L AM. Barriers to help-seeking by men: a review of sociocultural and clinical literature with particular reference to depression. J Affect Disord. 2002;71:1-9.

19. Hsu J, Donnelly JP, Moore JX, Meneses K. Williams G, Wang HE, National characteristics of emergency department visits by patients with cancer in the United States. Am J Emerg Med. 2018:36:2038-43. https://doi.org/10. 1016/j.ajem.2018.03.025. 
20. Mausbach BT, Irwin SA. Depression and healthcare service utilization in patients with cancer. Psychooncology. 2017;26:1133-9.

21. Guthrie EA, Dickens C, Blakemore A, Watson J, Chew-Graham C, Lovell K, et al. Depression predicts future emergency hospital admissions in primary care patients with chronic physical illness. J Psychosom Res. 2016;82:54-61. https://doi.org/10.1016/j.jpsychores.2014.10.002.

\section{Publisher's Note}

Springer Nature remains neutral with regard to jurisdictional claims in published maps and institutional affiliations.

Ready to submit your research? Choose BMC and benefit from:

- fast, convenient online submission

- thorough peer review by experienced researchers in your field

- rapid publication on acceptance

- support for research data, including large and complex data types

- gold Open Access which fosters wider collaboration and increased citations

- maximum visibility for your research: over $100 \mathrm{M}$ website views per year

At BMC, research is always in progress.

Learn more biomedcentral.com/submissions 